\title{
Effects of melatonin ingestion on cAMP and cGMP levels in human plasma
}

\author{
I V Zhdanova and D J Raz \\ Department of Brain and Cognitive Sciences, Massachusetts Institute of Technology, Cambridge, Massachusetts 02139, USA \\ (Requests for offprints should be addressed to I V Zhdanova, E18-439, Massachusetts Institute of Technology, Cambridge, Massachusetts 02139, USA; \\ Email: zhdanova@mit.edu)
}

\begin{abstract}
The effects of daytime melatonin treatment $(0 \cdot 3 \mathrm{mg})$ on cAMP and cGMP levels in platelet-rich plasma (PRP) and platelet-poor plasma (PPP) were investigated in 14 normal human subjects (age \pm S.E.M. 26.2 $\pm 3 \cdot 2$ years). Plasma levels of cAMP, cGMP and melatonin were measured before and at intervals for $3 \mathrm{~h}$ after the treatment was administered at $1300 \mathrm{~h}$.

Plasma melatonin concentrations reached peak levels $1 \mathrm{~h}$ after the treatment (mean \pm s.D. $182 \cdot 3 \pm 43 \cdot 5 \mathrm{pg} / \mathrm{ml}$ ). The mean areas under the curve (AUC) for the timecGMP concentration curves in PPP and in PRP were significantly increased after melatonin treatment compared with those observed after placebo treatment $(P=0 \cdot 001)$. No significant difference in cGMP levels was observed
\end{abstract}

between PPP and PRP. Increase in self-reported sleepiness after melatonin treatment positively correlated with increase in plasma cGMP levels $(r=0 \cdot 92)$. The mean AUC for the time-cAMP concentration in PRP, but not in PPP, was increased $1 \mathrm{~h}$ after melatonin treatment compared with that observed after placebo treatment, but not thereafter. No correlation between individual PRP or PPP cAMP levels and subjective sleepiness was observed. These results demonstrate a stimulating effect of melatonin treatment on plasma cGMP levels in humans and suggest a correlation between the increase in circulating cGMP levels and the sleep-promoting effect of the pineal hormone.

Journal of Endocrinology (1999) 163, 457-462

\section{Introduction}

In laboratory animals and in humans the pineal hormone melatonin is reported to influence several important physiological functions, including circadian rhythmicity, sleep and reproductive function (Lewy et al. 1996, Zhdanova et al. 1997, Reiter 1998). Melatonin receptors or binding sites have been identified in a variety of central neural sites and in peripheral tissues, e.g. the suprachiasmatic nuclei of the hypothalamus, the thalamus, the pars tuberalis of the pituitary gland, the retina and blood lymphocytes and platelets (Dubocovich 1984, Vanecek et al. 1987, Weaver et al. 1988, Williams \& Morgan 1988, Niles \& Hashemi 1990, Vacas et al. 1992).

The physiological functions and the mechanisms of melatonin's action in any of these diverse tissues remain obscure. However, in most of the tissues studied the hormone acutely inhibits intracellular cAMP accumulation through a G-protein coupled receptor (Reppert et al. 1994). The high lipid solubility of melatonin provides for the ready penetration of biological membranes by this amine, suggesting that its effects may be exerted not only at the membrane level but also intracellularly.

The marked diversity that has been observed in the behavioral effects of melatonin in different individuals
(Zhdanova \& Wurtman 1997) might be attributable not only to variations in its circulating concentration, but also to the relative sensitivity of different target cells to the pineal hormone. The concurrent assessment of both a behavioral and a cellular response to the hormone may facilitate understanding of the mode of action and interindividual variability in melatonin's effects. Since platelets were reported to be affected by melatonin treatment, we sought to examine the possibility that intracellular effects of melatonin in humans might be revealed by monitoring changes in the level of cyclic nucleotides in platelet-rich plasma (PRP) and platelet-poor plasma (PPP) samples obtained at intervals after the daytime administration of a low oral dose of melatonin $(0.3 \mathrm{mg})$ that tends to induce circulating melatonin levels within the physiological range (Dollins et al. 1994).

\section{Materials and Methods}

Fourteen healthy volunteers (13 males, 1 female; mean age \pm s.E.M. $26 \cdot 2 \pm 3 \cdot 2$ years) participated in a doubleblind placebo-controlled study after giving informed consent. The experimental protocol and the Subject's Informed Consent Form were approved by the 
Massachusetts Institute of Technology Committee on the Use of Humans as Experimental Subjects. Subjects underwent a physical examination and their urine and blood analyses were within the normal range. All of the subjects indicated that they were drug-free for at least 2 weeks prior to entering the study. They were asked to refrain from alcohol or caffeine consumption for $24 \mathrm{~h}$ prior to each test session, and to not take any medications during the entire period of the study.

Volunteers reported to the Clinical Research Center at $1000 \mathrm{~h}$ on the morning of each test session. A catheter with a saline lock was established in the subject's forearm vein $1 \mathrm{~h}$ before the first blood sample was collected and a light uniform lunch was served at noon. In test sessions, separated by at least 1 week without treatment, either a placebo (microcrystalline cellulose) or a $0.3 \mathrm{mg}$ dose of melatonin (Nestle Co., Vevey, Switzerland) was administered orally in a gelatin capsule at $1300 \mathrm{~h}$. The environmental light intensity was held constant on both test days at $250 \mathrm{~lx}$.

Blood samples, $6 \mathrm{ml}$ each, were drawn into polystyrene tubes containing $10 \%$ acid citrate dextrose anticoagulant solution (137 mM sodium citrate, $65 \mathrm{mM}$ citric acid, $104 \mathrm{mM}$ glucose). Blood samples were collected prior to treatment and at hourly intervals for $3 \mathrm{~h}$ after the treatment. The subjects were asked to report any changes in their subjective experience of sleepiness. Prior to each blood withdrawal, subjects were asked to report their sleepiness as either (1) decreased, (2) the same or (3) increased, relative to the pretreatment condition, on a three-score scale. Subjects were seated throughout the period of testing and they remained awake. Each of the subjects participated in two test sessions (at least a week apart) where they received either placebo or melatonin, half of the subjects receiving placebo first.

\section{PRP preparation}

PRP was obtained after blood samples were centrifuged at $200 \mathrm{~g}$ for $5 \mathrm{~min}$, 'low brake', at room temperature. Reactions were terminated immediately in two $100 \mu \mathrm{l}$ and two $200 \mu \mathrm{l}$ aliquots of PRP by the addition of $1 \mathrm{ml}$ ice-cold ethanol in order to measure baseline levels of cAMP and cGMP in the PRP. Triplicate $100 \mu \mathrm{l}$ aliquots of PRP were used to obtain platelet counts (Cell-DYN 3000, Abbott Diagnostics, Santa Fe Springs, CA, USA) and, thereby, to document the platelet concentration in each PRP sample. Platelet counts varied among the different subjects between 250 and $450 \times 10^{3} / \mu \mathrm{lPR}$, and were typically consistent in a set of replicate samples collected from the same subject. If we found more than a $10 \%$ difference between platelet counts in the same set of triplicate aliquots the results were not accepted and these PRP samples were not used to measure cyclic nucleotide concentrations.
After PRP samples were collected, the blood was further centrifuged for $5 \mathrm{~min}$ at $4000 \mathrm{~g}$, the plasma transferred into Eppendorff tubes, then centrifuged at $13000 \mathrm{~g}$ for $5 \mathrm{~min}$ to remove all remaining cells and yield PPP. Duplicate $100 \mu \mathrm{l}$ and $200 \mu \mathrm{lPP}$ aliquots were inactivated in $1 \mathrm{ml}$ ice-cold ethanol for cAMP and cGMP measurements. All inactivated PRP and PPP samples were centrifuged at $13000 \mathrm{~g}$, the supernatant was collected, lyophilized, reconstituted using the cAMP/cGMP assay buffer, and stored frozen at $-20{ }^{\circ} \mathrm{C}$ until assayed for cyclic nucleotides. A $500 \mu \mathrm{l}$ aliquot of each PPP sample was stored at $-20{ }^{\circ} \mathrm{C}$ until assayed for melatonin concentration.

In one subject, the variation among the platelet counts observed within the sets of triplicate aliquots of the samples collected in one of the test sessions repeatedly exceeded $10 \%$. For this reason his PRP data were excluded from the analysis and PRP results reflect data collected from 13 subjects only.

\section{Assays}

Both cAMP and cGMP concentrations in plasma samples were measured using $\left[{ }^{125} \mathrm{I}\right] \mathrm{cAMP}$ and $\left[{ }^{125} \mathrm{I}\right] \mathrm{cGMP}$ nonacetylation RIA kits (Amersham International, Amersham, Bucks, UK). Interassay coefficients of variation were less than $12.5 \%$. In the event that an intra-assay coefficient of variation exceeded $8 \%$, the assay was repeated. Standard cyclic nucleotide concentrations were repeatedly tested through each assay procedure in order to ensure the stable performance of the assay.

Plasma melatonin concentrations were also measured using a RIA kit (Buhlmann Laboratories, Allschwil, Switzerland). Melatonin extraction was accomplished using C18 columns. The limit of detection of the melatonin assay was $0.5 \mathrm{pg} / \mathrm{ml}$. The intra-assay coefficients of variation for control samples were $7 \cdot 5 \%(11 \mathrm{pg} / \mathrm{ml})$ and $7 \cdot 3 \%(25 \mathrm{pg} / \mathrm{ml})$; the corresponding interassay coefficients of variation were $12 \cdot 1$ and $15 \cdot 7 \%$.

Measurements of cAMP in PRP serially diluted with PPP established that platelet cAMP estimates (PPP cAMP concentration subtracted from PRP concentration) were proportional to the number of platelets counted. PPP samples, processed as described above, contained near zero platelets.

\section{Statistics}

Areas under the curves (AUC) for plasma cGMP and cAMP concentrations were calculated for two periods: a 3-h period after melatonin treatment and a $3-\mathrm{h}$ period after placebo treatment, from $1300 \mathrm{~h}$ to $1600 \mathrm{~h}$. A relationship between individual melatonin levels (peak level or AUC) or reported sleepiness (individual AUC for sleepiness scores over a 3-h period) and individual changes in cyclic nucleotide levels were analyzed using Pearson's correlation 


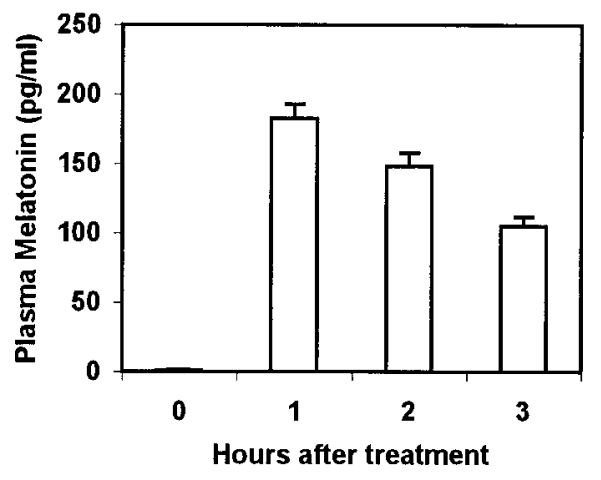

Figure 1 Group mean plasma melatonin levels (S.E.M.) for $3 \mathrm{~h}$ after ingestion of $0.3 \mathrm{mg}$ melatonin at $1300 \mathrm{~h}(n=14)$.

coefficient. For this comparison, changes in the individual cyclic nucleotide levels for each individual were expressed as AUC for the cyclic nucleotide concentration over a 3-h period, with each data point of such curves representing a difference between cyclic nucleotide levels at a particular time and a baseline level prior to ingestion of placebo or a $0.3 \mathrm{mg}$ dose of melatonin (delta AUC). Statistical comparison of peak melatonin levels after placebo or melatonin treatment, and of the AUC for plasma cGMP and cAMP after placebo or melatonin treatment, was performed using a two-tailed paired $t$-test. The minimum statistical significance level was $P<0 \cdot 05$. The results are presented as the means \pm s.E.M. Since cGMP levels in PRP and PPP were almost identical, and only 13 sets of data were available for PRP, all the statistical analysis was performed using PPP cGMP concentrations, which will be further referred to as plasma cGMP levels.

\section{Results}

Mean plasma melatonin levels before and $3 \mathrm{~h}$ after placebo treatment were $1.3 \pm 0.7$ and $1.6 \pm 0.8 \mathrm{pg} / \mathrm{ml}$ respectively. Peak melatonin levels were typically reached within $1 \mathrm{~h}$ after melatonin treatment; the mean melatonin level at this time was $182 \pm 10 \cdot 4 \mathrm{pg} / \mathrm{ml}$ (Fig. 1).

Plasma concentration of cAMP in PRP was significantly higher than in PPP at all time points tested, including baseline levels before placebo or melatonin treatment (PRP:1864 $\pm 52 \cdot 1$ and $1872 \pm 54 \cdot 4 \mathrm{fmol} / 100 \mu \mathrm{l}$; PPP: $916 \pm 76 \cdot 3$ and $932 \pm 79 \cdot 1 \mathrm{fmol} / 100 \mu \mathrm{l})$. cGMP levels in PRP and PPP were remarkably similar at baseline (PRP: $245 \pm 14 \cdot 3$ and $239 \pm 11 \cdot 2 \mathrm{fmol} / 100 \mu \mathrm{l}$; PPP: $246 \pm 17 \cdot 4$ and $251 \pm 15 \cdot 6 \mathrm{fmol} / 100 \mu \mathrm{l}$ ) and remained almost identical in PRP and PPP after placebo or melatonin treatment.

No significant changes in the concentration of both cyclic nucleotides were observed during a 3-h period after the placebo treatment. One hour after melatonin

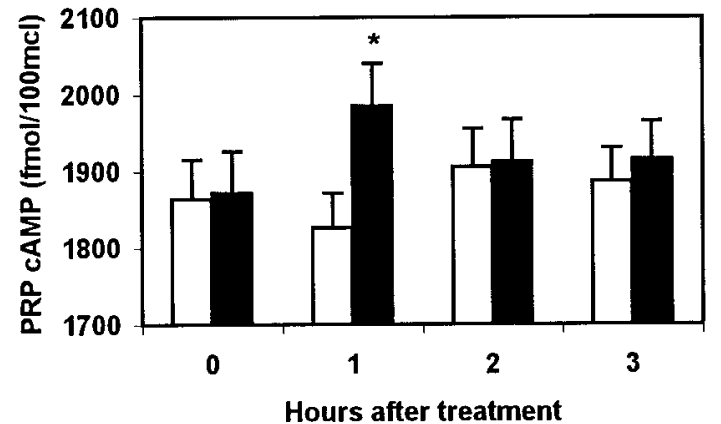

Figure 2 Group mean cAMP levels (S.E.M.) in PRP for $3 \mathrm{~h}$ after ingestion of placebo (white) or $0 \cdot 3 \mathrm{mg}$ melatonin (black) at $1300 \mathrm{~h} ;{ }^{*} P<0.05(n=13)$.

treatment PRP cAMP levels were transiently, but significantly $(P<0 \cdot 05)$, augmented (Fig. 2$)$. No changes in PPP cAMP levels were observed. Plasma cGMP concentration gradually increased during the 3-h period of testing following melatonin ingestion (Fig. 3). The mean AUC for the time-cGMP concentration curve was significantly increased after melatonin treatment compared with that observed after placebo treatment $(1210 \pm 223$ vs $995 \pm 226 \mathrm{fmol} / 100 \mu \mathrm{l}$; $P=0 \cdot 001)$. Individual changes in plasma cGMP or PRP cAMP levels did not correlate with the individual peak plasma melatonin levels or melatonin AUC after the hormone ingestion $(r=0 \cdot 31)$.

Placebo treatment did not cause a significant change in self-reported sleepiness until the third hour of testing. No significant correlation between cyclic nucleotide levels and sleepiness at the end of the placebo session was observed $(r=0 \cdot 27)$. After melatonin treatment, significant correlation was observed between the AUC of the increase in individual plasma cGMP levels relative to baseline (delta cGMP AUC) and sleepiness AUC ( $r=0.92)$ (Fig. 4). No such correlation was observed for PRP or PPP cAMP (PRP: $r=0 \cdot 22$ ). A significant increase in subjectively assessed sleepiness occurred $1 \mathrm{~h}$ after the hormone ingestion and $3 \mathrm{~h}$ after the administration of placebo (Fig. 3). No correlation was observed between individual baseline plasma cGMP or cAMP levels and the magnitude of a sleep-promoting effect of melatonin treatment or changes in plasma cGMP levels after the treatment.

\section{Discussion}

Our results demonstrate that the oral administration of melatonin to human adults, using a dose that increases the circulating melatonin concentration within the physiological range, substantially elevates the concentration of cGMP in plasma. The initiation of this increase can be detected $1 \mathrm{~h}$ after daytime melatonin treatment, coincident with the peak levels of circulating melatonin achieved. The rise in plasma cGMP concentrations 


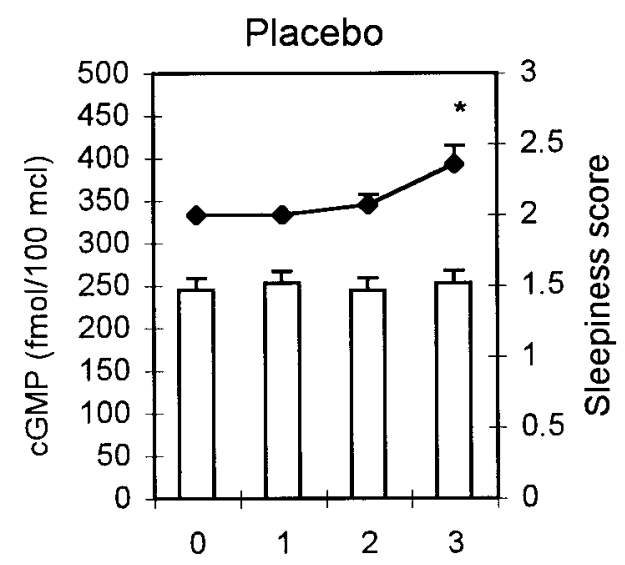

Hours after treatment

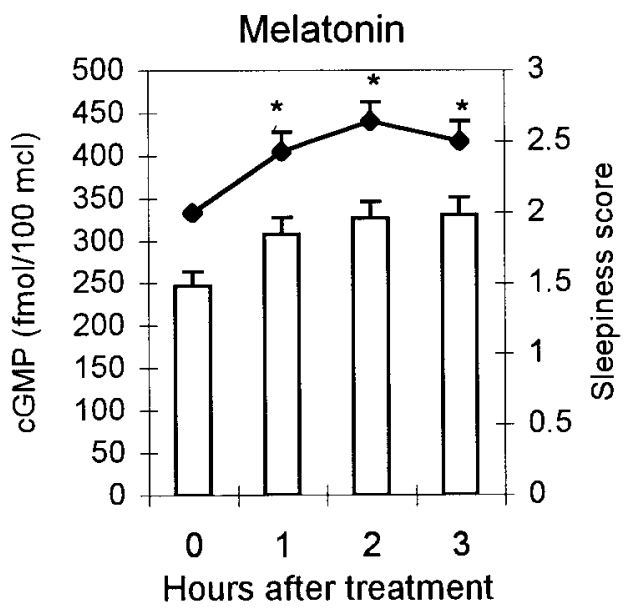

Figure 3 Columns: group mean cGMP levels (S.E.M.) in PPP for $3 \mathrm{~h}$ after ingestion of placebo or $0.3 \mathrm{mg}$ melatonin at $1300 \mathrm{~h}$. Lines: group mean sleepiness scores (S.E.M.) for $3 \mathrm{~h}$ after ingestion of placebo or $0.3 \mathrm{mg}$ melatonin at $1300 \mathrm{~h} ;{ }^{*} P<0.05(n=14)$.

continues for at least $2-3 \mathrm{~h}$ after melatonin is administered, while the circulating melatonin levels are gradually declining. The limited period of our observations precludes an estimate of the maximum duration of the cGMP stimulating effect. The period of increased plasma levels of cGMP after melatonin treatment and the magnitude of such increase positively correlated with the increase in subjectively reported sleepiness. The moderate increase in cAMP levels in PRP that was observed in some of the subjects within $1 \mathrm{~h}$ after melatonin treatment was not correlated with either the individual subject's sleepiness after the melatonin administration, nor with the magnitude of his peak circulating melatonin level.

The relationship between melatonin and cGMP levels has attracted the attention of a number of researchers. A 2to 3-fold increase in guanylate cyclase activity was observed in in vitro experiments involving the rat anterior
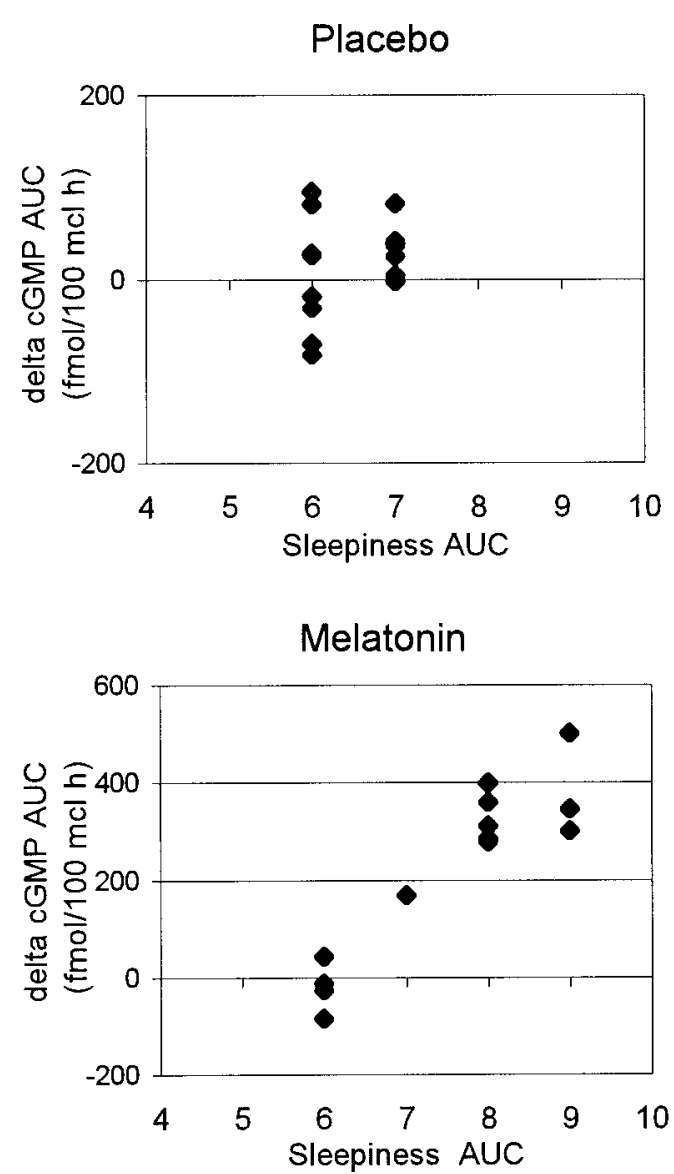

Figure 4 Relationship between individual $A \cup C$ of the increase in plasma cGMP relative to baseline (delta cGMP AUC) and sleepiness AUC for $3 \mathrm{~h}$ after ingestion of placebo or $0.3 \mathrm{mg}$ melatonin at $1300 \mathrm{~h}(n=14)$.

pituitary, thyroid, testis, ovary, liver and small intestine in the presence of a near physiological, $1 \mathrm{nM}$, melatonin concentration (Vesely 1981). Similarly, an increase in cGMP accumulation was reported in the rat medial basal hypothalamus (Vacas et al. 1981), in the hamster's retina (Faillace et al. 1996), and in murine mammary glands (Cardinali et al. 1992) incubated with melatonin. A significant increase in cGMP concentration in the cerebrospinal fluid (CSF) was found in both rabbits and humans after they were treated with high pharmacological doses of melatonin (Rudman 1976, Young et al. 1984).

Interestingly, while in both of these earlier CSF studies (Rudman 1976, Young et al. 1984) cAMP measurements were made, neither reported a significant effect of melatonin treatment on cAMP concentrations in CSF. We, similarly, did not observe any significant changes in PPP cAMP after the melatonin treatment. It suggests that if changes in the intracellular cAMP levels occur as a result of the in vivo melatonin treatment, they are not 
significantly reflected in such extracellular fluids as plasma or CSF. Though we have observed a clear increase in PRP cAMP levels $1 \mathrm{~h}$ after melatonin treatment in some of our subjects, the magnitude of the increase was modest. There are numerous observations in diverse tissues and brain structures studied in vitro that show an acute inhibition of cAMP accumulation as a result of melatonin treatment (Morgan et al. 1994). This effect is well described and linked to the hormone's interaction with the specific G-protein coupled receptors (Reppert et al. 1994). In contrast, an increase in cAMP accumulation was reported in other in vitro studies involving pars tuberalis cells (Hazlerigg et al. 1993) or rat Leydig cells (Valenti et al. 1995) after a prolonged, several hour, exposure of these tissues to melatonin. The differences in these observations may be attributable to a number of methodological considerations or possible downstream processes that may occur. A variability in the time course and in the magnitude of the adenyl cyclase suppression, its recovery and possible further sensitization after exposure to melatonin may be the crucial factors in the development of particular systemic and behavioral effects of the pineal hormone in vivo. An increase in cGMP concentration as a result of melatonin treatment could also affect the cAMP levels by modulating the phosphodiesterase- 3 activity. These results call for further detailed investigation of the dynamics of cAMP responses to melatonin treatment in in vivo experiments in animals and in human subjects.

The continued increase in concentrations of circulating cGMP observed in our subjects, following treatment with a physiological dose of melatonin, suggests that this effect might represent a normal physiological response to melatonin in humans. The increase in cGMP in the blood stream reflects an increased efflux of this intracellular second messenger. It may result from an increase in the intracellular cGMP production, an inhibition in its degradation, or an increase in its active release from the cells. It is not clear at this time specifically which cells might contribute to the observed phenomenon. As a first attempt to address the question of possible intracellular changes in in vivo cGMP concentrations we chose to test platelets, since an increase in cGMP concentration can reduce their activation (Waldmann \& Walter 1989) and melatonin was also shown to inhibit platelet aggregation (Vacas et al. 1991). However, our data did not reveal a significant difference in cGMP levels between PRP and PPP, independent of the treatment administered. Thus, platelets do not substantially accumulate cGMP under the conditions tested. It is possible, though, that an increased intracellular cGMP production in platelets was compensated for by an increased cGMP efflux, as it has been shown in platelet activation studies (Wu et al. 1993).

The fact that that there was a significant positive correlation between an increase in subjective sleepiness after melatonin treatment and an increase in cGMP concentrations suggests that these two effects of melatonin might be related. Such a hypothesis is further substantiated by our recent finding that there is a significant daily variation in human plasma cGMP levels with an increase concurrent with the habitual nighttime hours of sleep (Zhdanova et al. 1999). Further studies involving larger numbers of human subjects, and studies in other diurnal animals that are sensitive to sleep-promoting effects of melatonin (Zhdanova et al. 1998), are needed in order to establish and explore the peripheral and central mechanisms of this possible relationship between the molecular and behavioral parameters of melatonin's action.

\section{Acknowledgements}

We would like to thank Harry J Lynch for useful discussions and the Massachusetts Institute of Technology Undergraduate Research Opportunity Program for their help. This work was supported in part by grants from the National Institutes of Health (1RO1 AG13667-01, MO1 RR 02172 and MO1 RR 00088-34) and the Center for Brain Sciences and Metabolism Charitable Trust.

\section{References}

Cardinali DP, Bonanni Rey RA, Mediavilla MD \& Sanchez-Barcelo E 1992 Diurnal changes in cyclic nucleotide response to pineal indoles in murine mammary glands. Journal of Pineal Research 13 111-116.

Dollins AB, Zhdanova IV, Wurtman RJ, Lynch HJ \& Deng MH 1994 Effect of inducing nocturnal serum melatonin concentrations in daytime on sleep mood body temperature and performance. Proceedings of the National Academy of Sciences of the USA 91 1824-1828.

Dubocovich ML 1984 Melatonin is a potent modulator of dopamine release in the retina. Nature $306782-784$.

Faillace MP, Keller Sarmiento MI \& Rosenstein RE 1996 Melatonin effect on the cyclic GMP system in the golden hamster retina. Brain Research 711 112-117.

Hazlerigg DG, Gonzalez-Brito A, Lawson W, Hastings MH \& Morgan PJ 1993 Prolonged exposure to melatonin leads to time-dependent sensitization of adenylate cyclase and downregulates melatonin receptors in pars tuberalis cells from ovine pituitary. Endocrinology 132 285-292.

Lewy AJ, Ahmed S \& Sack RL 1996 Phase shifting the human circadian clock using melatonin. Behavior and Brain Research $\mathbf{7 3}$ 131-134.

Morgan PJ, Barrett P, Howell HE \& Helliwell R 1994 Melatonin receptors: localization, molecular pharmacology and physiological significance. Neurochemistry International 24 101-146.

Niles LP \& Hashemi F 1990 Picomolar-affinity binding and inhibition of adenylate cyclase activity by melatonin in Syrian hamster hypothalamus. Cellular and Molecular Neurobiology 10 553-558.

Reiter RJ 1998 Melatonin and human reproduction. Annals of Medicine 30 103-108.

Reppert SM, Weaver DR \& Ebisawa T 1994 Cloning and characterization of a mammalian melatonin receptor that mediates reproductive and circadian responses. Neuron 13 1177-1185.

Rudman D 1976 Injection of melatonin into cisterna magna increases concentration of $3^{\prime}, 5^{\prime}$ cyclic guanosine monophosphate in cerebrospinal fluid. Neuroendocrinology $20235-242$. 
Vacas MI, Sarmiento MI \& Cardinali DP 1981 Melatonin increases cGMP and decreases cAMP levels in rat medial basal hypothalamus in vitro. Brain Research 225 207-211.

Vacas MI, Del Zar MM, Martinuzzo M, Falcon C, Carreras LO \& Cardinali DP 1991 Inhibition of human platelet aggregation and thromboxane B2 production by melatonin. Correlation with plasma melatonin levels. Journal of Pineal Research 11 135-139.

Vacas MI, Del Zar MM, Martinuzzo M \& Cardinali DP 1992 Binding sites for -melatonin in human platelets. Journal of Pineal Research 13 60-65.

Valenti S, Guido R, Giusti M \& Giordano G 1995 In vitro acute and prolonged effects of melatonin on purified rat Leydig cell steroidogenesis and adenosine $3^{\prime}, 5^{\prime}$-monophosphate production. Endocrinology 136 5357-5362.

Vanecek J, Pavlik A \& Illnerova H 1987 Hypothalamic melatonin receptor sites revealed by autoradiography. Brain Research 435 359-362.

Vesely D 1981 Melatonin enhances guanylate cyclase activity in a variety of tissues. Molecular and Cellular Biochemistry 35 55-58.

Waldmann R \& Walter U 1989 Cyclic nucleotide elevating vasodilators inhibit platelet aggregation at an early step of the activation cascade. European Journal of Pharmacology $\mathbf{1 5 9}$ 317-20.

Weaver DR, Namboodiri MA \& Reppert SM 1988 Iodinated melatonin mimics melatonin action and reveals discrete binding sites in fetal brain. FEBS Letters 228 123-127.
Williams LM \& Morgan PJ 1988 Demonstration of melatonin-binding sites on the pars tuberalis of the rat. Journal of Endocrinology 119 R1-R3.

Wu XB, Brune B, Von Appen F \& Ullrich V 1993 Efflux of cyclic GMP from activated human platelets. Molecular Pharmacology 43 564-568.

Young S, Gauthier S, Kiely ME, Lal S \& Brown GM 1984 Effect of oral melatonin administration on melatonin, 5-hydroxyindoleacetic acid, indoleacetic acid, and cyclic nucleotides in human cerebrospinal fluid. Neuroendocrinology 39 87-92.

Zhdanova IV \& Wurtman RJ 1997 Efficacy of melatonin as a sleep-promoting agent. Journal of Biological Rhythms 12 644-650.

Zhdanova IV, Lynch HJ \& Wurtman RJ 1997 Melatonin: a sleep promoting hormone. Sleep 20 899-907.

Zhdanova IV, Cantor ML, Leclair OU, Kartashov AI \& Wurtman RJ 1998 Behavioral effects of melatonin treatment in non-human primates. Sleep Research Online 1 114-118.

Zhdanova IV, Marcus JN, Raz DJ, Busza AC, Leclair OU \& Wurtman RJ 1999 Daily variation in plasma cGMP and effects of melatonin treatment. Sleep 22 (Suppl 1) S4.

Received 3 December 1998

Revised manuscript received 31 May 1999

Accepted 27 July 1999 